\title{
In-Situ S/TEM and In-Situ SIMS Multi-Modality Probing of Chemical and Structural Evolution of Rechargeable Battery
}

\author{
Chongmin Wang
}

Environmental Molecular Sciences Laboratory, Pacific Northwest National Laboratory, Richland, WA 99354, USA.

Repeated charging and discharging of the Li-ion battery induces microstructural evolutions both at the interface between the electrolyte and the electrode and within the electrode. Although it has been established that this microstructural evolution is responsible for the failure of the battery, the mechanisms of the microstructural changes as a function of charging/discharging are not well understood. In this presentation, I will highlight recent progress on ex-situ, in-situ and operando S/TEM [1-3] and in-situ liquid SIMS [4] for probing into the structural and chemical evolution of energy storage materials. In perspective, challenges and possible direction for further development of the in-situ S/TEM imaging and spectroscopic methods for energy storage materials and other field will also be discussed.

For in-situ S/TEM, a miniature prototype battery was built using $\mathrm{LiCoO}_{2}$ as the cathode, $\mathrm{SnO}_{2}$ nanowire as anode and an ionic liquid electrolyte. The cathode was a mixture of $\mathrm{LiCoO}_{2}$ particles, conductive carbon and PVDF binder, and coated as a thin film on an aluminum foil. The anode was $\mathrm{SnO}_{2}$ nanowire, which gives the advantage of electron transparency and therefore allows in-situ observation of both chemical and structural change under TEM during the operation of the battery. The electrolyte was air stable where the salt lithium bis(trifluoromethane sulfonyl)imide (LiTFSI) was dissolved in a hydrophobic ionic liquid 1-butyl-1-methylpyrrolidium $\left(\mathrm{P}_{14}\right)$ bis(trifluoromethane sulfonyl)imide ( $\mathrm{P}_{14}$ TFSI). The overall composition of the electrolyte was $10 \%$ LiTFSI in $\mathrm{P}_{14}$ TFSI. It has been tested that this ionic-liquid-based electrolyte is stable in the column of the TEM with a typical vacuum of $10^{-5}$ $\mathrm{Pa}$ and shows no significant evaporation following one week in the TEM column, therefore allowing a systematic cyclic charging and discharging of the battery in the TEM. The overall designing of the battery was shown in Figure 1.

In-situ TEM observations provide vivid morphological information on the evolution of electrode surface. However, the chemical composition and structure of the solid electrolyte interphase (SEI) layer cannot be directly captured by imaging through the liquid layers in the setup of the in-situ TEM. Furthermore, questions about the specific species migrating within the liquid electrolyte and the ways they interact with the electrode cannot be readily answered by in-situ TEM. To address these questions, we describe the creation of liquid-cell secondary ion mass spectrometry (SIMS) approach for in-situ or more precisely operando SIMS studies of Li-ion batteries, gaining molecular level information that evolves at both the electrode surface and in the liquid electrolyte during the charge and discharge of the battery (Figure 1).

The fundamental concept of the liquid SIMS device is illustrated in Figure 1. In essence, the liquid to be analyzed is exposed by creating a small hole into which a probing primary beam can interact with the liquid. For the rechargeable battery, an electrode created by coating $\mathrm{LiCoO}_{2}$ powder particles on $\mathrm{Al}$ foil was used as cathode. The anode side was fabricated on a silicon chip with a "transparent" $\mathrm{Si}_{3} \mathrm{~N}_{4}$ membrane window of $100 \mathrm{~nm}$ in thickness and lateral dimensions of $0.5 \mathrm{~mm} \times 0.5 \mathrm{~mm}$. A Cu film of 
$70 \mathrm{~nm}$ was sputter deposited on the $\mathrm{Si}_{3} \mathrm{~N}_{4}$ film/window. An electrolyte comprised of $1.0 \mathrm{M} \mathrm{LiPF}_{6}$ in 1:2 v/v ethylene carbonate:dimethyl carbonate ( $\mathrm{LiPF}_{6}$ in $\left.\mathrm{EC}: \mathrm{DMC}\right)$ was used.

The in-situ liquid SIMS analysis indicate that, upon charging of the battery, the salt anions (hexafluorophosphate $\left(\mathrm{PF}_{6}{ }^{-}\right)$anions in this work) were repelled from the anode side, $\mathrm{Li}^{+}$ions were reduced at the anode, leading to a liquid layer with a significantly low concentration of $\mathrm{Li}^{+}$and $\mathrm{PF}_{6}^{-}$ around the anode. Formation of the lean electrolyte layer around the electrode will lead to reduced ionic conductivity and therefore contributing to the overpotential of the battery.

The present work clearly illustrate recent development on the in-situ S/TEM and in-situ liquid SIMS as a technique and their application for probing detailed structural and chemical evolution of rechargeable battery. Most importantly, we also demonstrated that integration of different instrumentations is critical for capturing complementary information [5].

\section{References:}

[1] CM Wang, J Mater Res 30 (2015), p. 326.

[2] JY Huang et al, Science 330 (2010), p. 1515.

[3] M Gu et al, Nano Lett 13 (2013), p. 6106.

[4] Z Zhu et al, Nano Lett 15 (2015),p. 6170.

[5] This work was supported by Battery Materials Research, DOE. The work was conducted in the William R. Wiley Environmental Molecular Sciences Laboratory (EMSL), a national scientific user facility sponsored by DOE's Office of Biological and Environmental Research and located at PNNL.

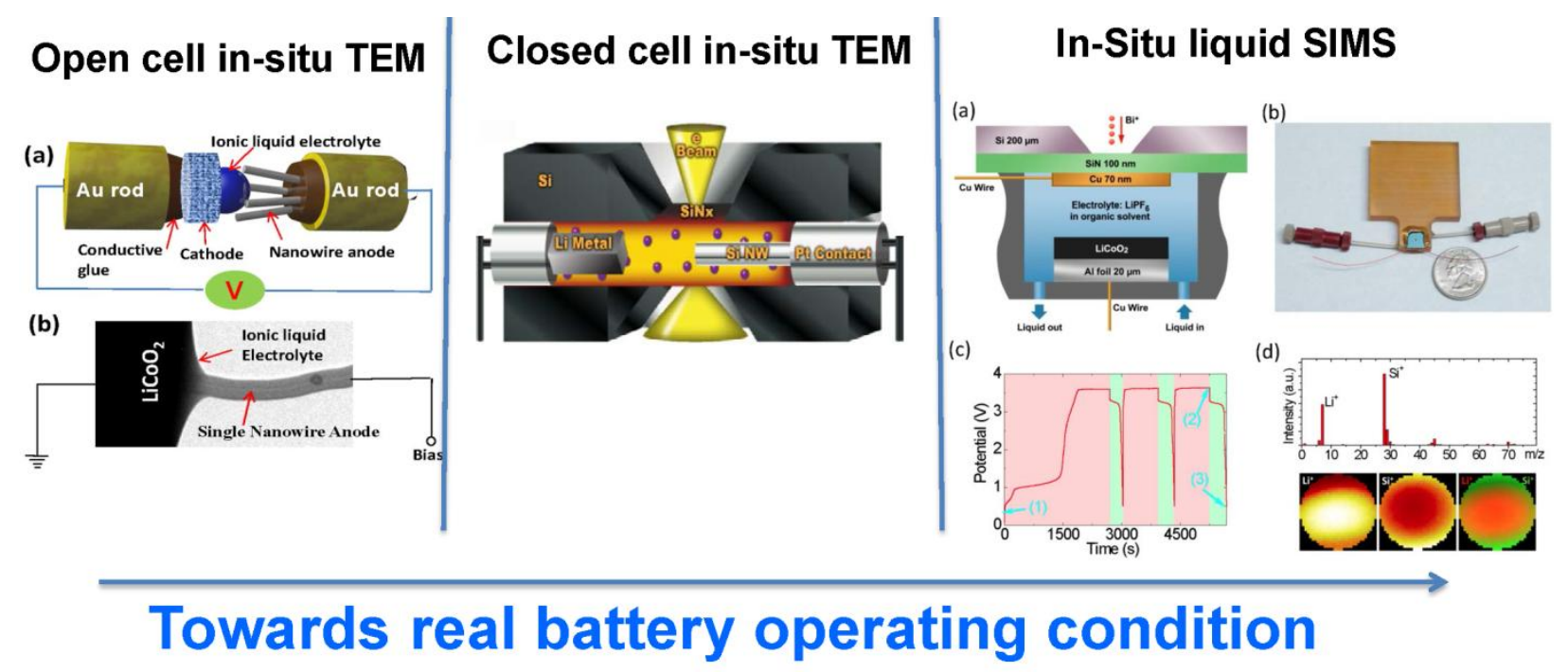

Figure 1. Fundamental concept on the designing of prototype lithium ion battery for in-situ TEM and in-situ liquid SIMS study of rechargeable battery under operating condition [1], with permission from Cambridge University Press. 